\title{
Erradicação do Helicobacter pylori: fim de linha para a terapêutica tripla standard?
}

António Assunção Silva, ${ }^{1}$ Rui Macedo, ${ }^{2}$ Dina Novais Fernandes, ${ }^{3}$ Luís Sousa, ${ }^{3}$ Adriana Miranda ${ }^{4}$

\section{RESUMO}

Objetivo: Rever a evidência sobre a eficácia da terapêutica tripla (TT) e da terapêutica sequencial (TS) na erradicação da infeção por Helicobacter pylori (H. pylori).

Fontes de Dados: MEDLINE e sítios de medicina baseada na evidência.

Métodos: Pesquisa de normas de orientação clínica (NOC), revisões sistemáticas (RS), meta-análises (MA) e ensaios clínicos aleatorizados e controlados (EAC), publicados entre junho/2004 e junho/2014, utilizando os termos MeSH Helicobacter pylori, Drug therapy e Disease eradication. Para atribuição do nível de evidência (NE) e da força de recomendação (FR) foi utilizada a Strenght of Recomendation Taxonomy (SORT).

Resultados: Foram selecionados 15 de entre 227 artigos encontrados: três NOC, oito MA, duas RS e dois ECA. As NOC recomendam a TT como principal tratamento a ser utilizado. As oito MA e as duas RS evidenciaram superioridade da TS na erradicação do H. pylori (variando entre 81-95,6\%) comparativamente à TT (principalmente de 7-10 dias, consistentemente <80\%). Os dois EAC obtiveram dados sobreponíveis às MA e RS.

Conclusões: A TS apresenta uma eficácia superior comparativamente à TT e deve ser considerada, atualmente, como terapêutica de 1. ' linha na erradicação da infeção por $H$. pylori (FR A). Se comparada com a TT-14d, a TS convencional parece apresentar taxas de erradicação sobreponíveis embora, quando aumentada para 14 dias, a TS apresente superioridade terapêutica (FR B). Nas áreas de elevada resistência à claritromicina, metronidazol ou ambos, como é o caso de Portugal, aumenta a superioridade da TS face à TT (FR A).

Termos MeSH: Helicobacter pylori; Drug Therapy; Disease Eradication.

\section{INTRODUÇÃO}

A infeção por Helicobacter pylori (H. pylori) é certamente a mais comum a nível global, prevendo-se que metade da população mundial esteja infetada. ${ }^{1}$ A sua tendência para causar doença e morte é uma preocupação ao nível da saúde pública em todo o mundo. ${ }^{1-2}$ É a principal causa de gastrite crónica, úlcera péptica, adenocarcinoma gástrico e linfoma MALT gástrico. ${ }^{3}$

O principal objetivo terapêutico na infeção por $H$. pylorié a sua erradicação completa. Por conseguinte, a eficácia do regime terapêutico deve ser o principal fator decisivo aquando da sua prescrição. ${ }^{1}$ As principais guidelines ainda recomendam a terapêutica tripla (TT) convencional [inibidor da bomba de protões (IBP) + claritromicina + amoxicilina ou metronidazol, durante 7-14 dias] como tratamento de $1 .{ }^{a}$ linha. ${ }^{24-6}$

${ }^{1}$ Médico interno de Medicina Geral e Familiar. na USF MaxiSaúde, Braga. ${ }^{2}$ Médico Especialista em Medicina Geral e Familiar e orientador de formação na USF MaxiSaúde, Braga.

${ }^{3}$ Médicos internos de Medicina Geral e Familiar na USF +Carandá, Braga. ${ }^{4}$ Médica interna de Medicina Geral e Familiar na USF Pró-Saúde, Vilaverde.
No entanto, a prevalência da resistência deste agente patogénico à claritromicina e ao metronidazol aumentou substancialmente nos últimos anos. Já na década de 90, num estudo realizado por Cabrita et al. verificou-se que, em Portugal, nomeadamente no distrito de Lisboa, as taxas de resistência ao metronidazol e à claritromicina eram de $30,6 \% \mathrm{e}$ $19,0 \%$, respetivamente. ${ }^{7}$ Um estudo mais recente, publicado em maio de 2012, revelou uma taxa de resistência à claritromicina de $31,3 \% \mathrm{em}$ Portugal, sendo este o $3 .^{\circ}$ maior valor da Europa, logo a seguir à Áustria e Hungria. ${ }^{8}$

O aumento das resistências conduziu, inevitavelmente, a uma diminuição da taxa de erradicação do H. pylori, a qual declinou para valores insatisfatórios em Portugal, ${ }^{9}$ bem como na maioria dos países ocidentais, ${ }^{10}$ encontrando-se agora, comummente, abaixo dos $80 \%{ }^{8,11}$ Nos EUA, as taxas de erradicação rondam os 75\%;"12-13 na Rússia e na Malásia os 60\%;" e em Portugal, Alemanha, Islândia e Turquia rondam os 50\%. ${ }^{9}$ Por este motivo é, hoje, globalmente aceite que novas estratégias são necessárias para o seu tratamento. ${ }^{2-3,11}$

A terapêutica sequencial (TS) tem demonstrado resulta- 
dos promissores comparativamente com a TT,,$^{1-3,14-15}$ quer em adultos quer em idade pediátrica. ${ }^{2} \mathrm{Na} \mathrm{TS}$, os fármacos efetivos contra a bactéria são utilizados numa combinação diferente, sendo o regime sequencial mais frequentemente utilizado o que consta de IBP + amoxicilina nos primeiros cinco dias, seguido de IBP + claritromicina + metronidazol ou tinidazol ${ }^{3,11,14,16}$ ou amoxicilina ${ }^{1}$ nos cinco dias seguintes.

Esta revisão tem como objetivo rever a evidência disponível sobre a eficácia da TS na erradicação do H. pylori, comparativamente com a TT convencional.

\section{MÉTODOS}

Foi realizada uma pesquisa de normas de orientação clínica (NOC), ensaios clínicos aleatorizados e controlados (ECA), revisões sistemáticas (RS) e meta-análises (MA), publicados entre junho de 2004 e junho de 2014, em português, inglês e espanhol, nas bases de dados da National Guideline Clearing House, Guidelines Finder, CMA Infobase, Cochrane Library, DARE, Bandolier, Evidence Based Medicine Online, TRIP Database e MEDLINE, utilizando os termos MeSH: Helicobacter pylori, Drug therapye Disease eradication. Os critérios de inclusão dos artigos consistiram em: população alvo constituída por adultos ou crianças com diagnóstico de infeção por H.pylori estabelecido por, pelo menos, um de entre o exame histológico, teste rápido da urease, teste respiratório da ureia ou pesquisa de antigénio fecal do $H$. pylori; intervenção terapêutica com TS com dois períodos de tratamento; em comparação com a TT convencional; e outcome baseado na taxa de eficácia da erradicação do H. pylori, avaliada por teste de confirmação. Foram utilizados como critérios de exclusão: estudos que incluíssem doentes com falência no tratamento prévio para a erradicação do H. pylori ou a realizar outra terapêutica antibiótica concomitante; artigos duplicados; artigos de opinião; artigos de revisão clássica de tema; e artigos discordantes com o objetivo da revisão.

Para estratificar o nível de evidência (NE) dos estudos e a força de recomendação (FR) foi utilizada a Strenght of Recomendation Taxonomy (SORT), da American Academy of Family Physicians. ${ }^{17}$

\section{RESULTADOS}

A pesquisa efetuada resultou na identificação de 227 artigos e na seleção de 15 que respeitavam todos os critérios de inclusão e de exclusão: três NOC, dois ECA, duas RS e oito MA. A Figura 1 representa o fluxograma da seleção dos estudos.

As principais NOC atuais - de Maastricht,$^{4}$ da American College of Gastroenterology $(\mathrm{ACG})^{6} \mathrm{e}$ da Asia-Pacific guide- lines $^{5}$ - recomendam a TT como principal tratamento a ser utilizado, a nível mundial, para a erradicação do $H$. pylori (Quadro I). Esta consiste na associação de IBP 2id + claritromicina 500mg 2id + amoxicilina 1g 2id ou metronidazol 500mg 2id, durante 7-14 dias. As duas primeiras NOC recomendam a TS como terapêutica alternativa, embora a de Maastricht restrinja essa indicação às áreas com taxa de resistência à claritromicina superior a $15-20 \%$, se os sais de bismuto para terapêutica quádrupla não estiverem disponíveis (FR A). ${ }^{4}$ Quer a Asia-Pacific Guidelines quer a ACG relatam a necessidade de validação da TS, através de mais estudos multicêntricos, randomizados e de boa qualidade antes de poderem recomendar a TS como terapêutica de $1 .{ }^{\mathrm{a}}$ linha na erradicação do H. pylori (FR C). ${ }^{5-6}$

Kim et al. realizaram, em 2014, uma MA (Quadro II) em que foram incluídos nove ECA, todos asiáticos. ${ }^{3}$ Nesta, a TS revelou-se superior à TT de 7, 10 e 14 dias, embora sem significância estatística no último grupo $(p=0,000, p=0,004 \mathrm{e}$ $p=0,148$, respetivamente). A taxa de erradicação agrupada da TS por análise Intention-to-treat (ITT) e Per Protocol (PP) $(81,3 \%$ e $87,6 \%)$ foi superior à da TT $(70,8 \%$ e $77,1 \%)$, assim como o valor de odds-ratio (OR) na análise ITT e $P P(1,768$ e 1,997, respetivamente, ambos com $p<0,001)$. Em três dos estudos utilizou-se tinidazol e, nos restantes, metronidazol como parte da TS revelando, ambos os esquemas, superioridade face à TT. Não se observaram diferenças entre as duas terapêuticas quanto aos efeitos laterais. Os autores concluíram que a TS parece ser superior à TT, pelo que pode ser considerada uma opção como tratamento de $1 .{ }^{\text {a }}$ linha.

A MA de Gatta et al., publicada em 2013 (Quadro II), incluiu $43 \mathrm{ECA}$, sendo que se comparou a eficácia da TS com a TT-7d em 22 deles, com a TT-10d em 14 e com a TT-14d em sete estudos..$^{11} \mathrm{~A}$ taxa de erradicação da TS foi superior à TT-7d e TT-10d [risco relativo $(\mathrm{RR})=1,21$ e 1,11 , respetivamente], mas não comparativamente com a TT-14d $(R R=0,99)$. Em dois dos estudos comparou-se a TS-10d com a TS-14d, não tendo sido constatada diferença estatisticamente significativa entre ambos os regimes. Nos indivíduos infetados por estirpes de H. pylori resistentes à claritromicina e ao metronidazol, detetados por testes de sensibilidade antimicrobiana pré-tratamento, a TS obteve melhores taxas de erradicação, mas apenas em comparação com a TT de sete e de 10 dias. A taxa de erradicação global com a TS foi de $84,3 \%$, o que levou os autores a concluir que as taxas de erradicação são subótimas com qualquer dos regimes terapêuticos. Não se observaram diferenças entre as duas terapêuticas quanto aos efeitos laterais. 


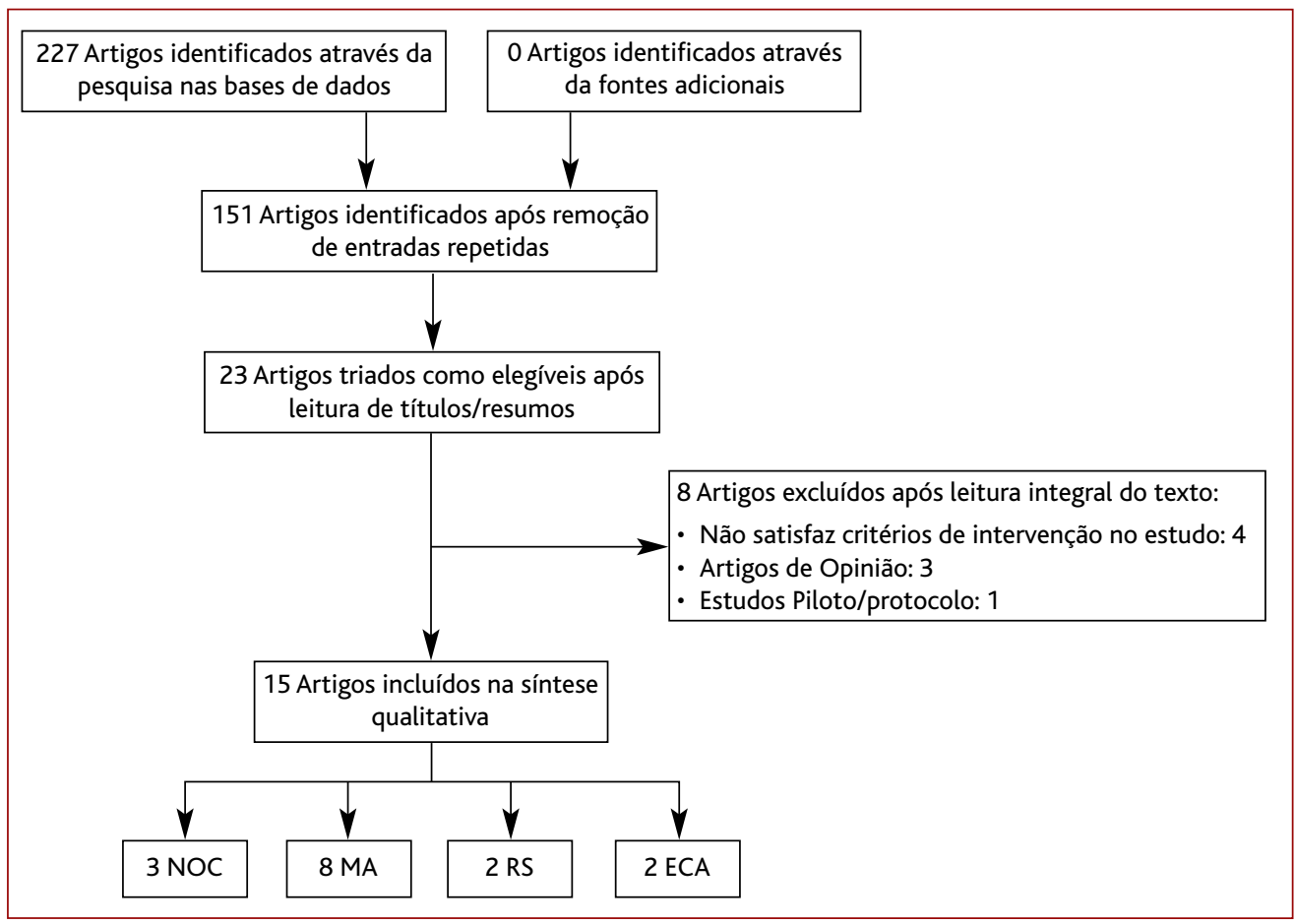

Figura 1. Fluxograma de seleção dos estudos, segundo as normas PRISMA.

do estatisticamente significativo ter sido alcançado apenas na comparação com a TT-7d (12\% mais eficaz, com $p=$ 0,014). A taxa de erradicação global ascendeu aos $91,0 \%$ nos adultos e a $90,7 \%$ nas crianças com a TS, ficando pelos $75,7 \%$ e $82,9 \%$, respetivamente, com a TT. Nos casos detetados de infeção por estirpes de $H$. $p y$ lori resistentes à claritromicina, a diferença na eficácia da erradicação entre os dois regimes dilatou-se, favorecendo a TS (83,3\% vs. 25,9\%, com $O R=10,21) .{ }^{18-19}$ Os autores concluíram que a TS é uma terapia promissora, aparentemente supe-

Outra MA de Gatta et al., de 2009, incluiu 10 ECA envolvendo 3.006 adultos e três ECA envolvendo 260 crianças e adolescentes (Quadro II). ${ }^{2}$ Do total dos estudos, 10 foram realizados em Itália e os restantes na Roménia, China e Coreia. Em ambos os grupos etários, a TS foi superior em relação à TT (adultos: $O R$ agrupado=2,99; crianças e adolescentes: $O R$ agrupado=1,98), apesar de, no grupo das crianças, o resulta- rior à TT na erradicação do H. pylori. Todavia, alegaram a necessidade de mais estudos de elevada qualidade antes de poder ser recomendada como terapêutica de $1 .^{\mathrm{a}}$ linha.

Na MA deYoon etal., publicada em 2013 (Quadro II), foram incluídos 17 ECA, todos realizados em países asiáticos. ${ }^{14} \mathrm{~A}$ taxa de erradicação, por análise ITT, foi significativamente superior na TS $(81,8 \%)$ em comparação com a

\section{QUADRO I. Normas de Orientação Clínica}

\begin{tabular}{|c|c|c|c|}
\hline Referência & Ano & Recomendações & NE \\
\hline $\begin{array}{l}\text { Maastricht IV/Florence } \\
\text { Consensus Report }\end{array}$ & 2012 & $\begin{array}{l}\text { A TT standard (1. a linha) deve ser evitada em áreas com resistência à claritromicina } \\
>15-20 \% \text { (FR D). } \\
\text { Em áreas de resistência elevada à claritromicina, a terapêutica sequencial é recomendada } \\
\text { quando a terapêutica quádrupla contendo bismuto não for possível (FR A). }\end{array}$ & 33 \\
\hline $\begin{array}{l}\text { Asia-Pacific Consensus } \\
\text { Guidelines }^{5}\end{array}$ & 2009 & 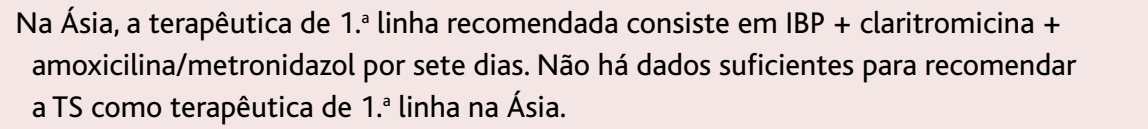 & 33 \\
\hline $\begin{array}{l}\text { American College of } \\
\text { Gastroenterology }\end{array}$ & 2007 & $\begin{array}{l}\text { TS pode constituir uma alternativa ao esquema TT standard (IBP + claritromicina + } \\
\text { amoxicilina ou metronidazol) ou terapêutica quádrupla com sais de bismuto, mas } \\
\text { requer validação antes de ser recomendado como terapêutica de } 1 .^{\text {a linha. }}\end{array}$ & 33 \\
\hline
\end{tabular}

Legenda: TT - Terapêutica tripla; TS - Terapêutica sequencial; IBP - Inibidor da bomba de protões; FR - Força de recomendação. 


\begin{tabular}{|c|c|c|c|c|}
\hline Referência & $\begin{array}{l}\text { Estudos } \\
\text { incluídos }\end{array}$ & Intervenção & Resultados & NE \\
\hline $\begin{array}{l}\text { Kim et al., } \\
2014^{3}\end{array}$ & $\begin{array}{l}9 \text { ECA } \\
n=3.074\end{array}$ & $\begin{array}{l}\text { TS } 5 d+5 d \text { vs. TT- } 7 d \\
\text { (5 ECA), TT-10d (3 ECA) } \\
\text { ou TT-14d (3 ECA) }\end{array}$ & $\begin{array}{l}\text { A TS é mais efetiva do que a TT-7d }(O R=1,79 ; p<0,001) \text { e } \\
\text { 10d (OR 1,65; } p=0,004) ; \text { no entanto, não se revelou superior } \\
\text { à TT-14d }(O R 1,45 ; p=0,148) \text {. }\end{array}$ & 1 \\
\hline $\begin{array}{l}\text { Gatta et al., } \\
2013^{11}\end{array}$ & $\begin{array}{l}43 \mathrm{ECA} \\
n=13.532\end{array}$ & $\begin{array}{l}\text { TS } 5 d+5 d \text { vs. TT-7d } \\
\text { (22 ECA), TT-10d (14 ECA) } \\
\text { ou TT-14d (7 ECA) }\end{array}$ & $\begin{array}{l}\text { TS superior na erradicação do } H \text {. pylori em comparação com } \\
\text { TT-7d }(R R=1,21) \text { e TT-10d ( } R R=1,11) \text {, mas não } \\
\text { comparativamente com TT-14d }(R R=0,99) \text {. }\end{array}$ & 2 \\
\hline $\begin{array}{l}\text { Yoon et al., } \\
2013^{14}\end{array}$ & $\begin{array}{l}17 \text { ECA } \\
n=3.419 \\
\text { (Adultos } \\
\text { Asiáticos) }\end{array}$ & $\begin{array}{l}\text { TS } 5 d+5 d \text { vs. TT-7d } \\
\text { (10 ECA), TT-10d (5 ECA) } \\
\text { ou TT-14d (4 ECA) }\end{array}$ & $\begin{array}{l}\text { TS é superior à TT em termos de eficácia na erradicação do } \\
\text { H. pylori (ITT: } 81,8 \%, 95 \% \text { IC: } 78,9-84,6) \text {, mesmo assim } \\
\text { ligeiramente abaixo do desejável (ITT } \geq 90 \%) \text {. }\end{array}$ & 2 \\
\hline $\begin{array}{l}\text { Chung et al., } \\
2013^{22}\end{array}$ & $\begin{array}{l}6 \text { ECA } \\
n=1.529 \\
\text { (Adultos } \\
\text { Coreanos) }\end{array}$ & $\begin{array}{l}\text { TS } 5 d+5 d \text { vs. TT-7d } \\
\text { (3 ECA), TT-10d (3 ECA) } \\
\text { ou TT-14d (2 ECA) }\end{array}$ & $\begin{array}{l}\text { TS é superior à TT em termos de eficácia na erradicação do } \\
\text { H. pylori } \\
\text { ITT: } 79,7 \text { vs. } 68,1 \% \text {; OR } 1,838(p<0,001) \text {; } \\
\text { PP: } 86,4 \% \text { vs. } 76,0 \% \text {; OR } 1,974(p<0,001) \text {. }\end{array}$ & 2 \\
\hline $\begin{array}{l}\text { Horvath, } \\
\text { Dziechciarz, } \\
\text { Szajewska, } \\
2012^{23}\end{array}$ & $\begin{array}{l}10 \text { ECA } \\
n=857 \\
\text { crianças } \\
\text { (3-18 anos) }\end{array}$ & $\begin{array}{l}\text { TS } 5 d+5 d \text { vs. TT- } 5 d \\
\text { (TT-1 ECA), } 7 d(5 \text { ECA), } \\
\text { TT-10d ( } 2 \text { ECA) ou } \\
\text { TT-14d (3 ECA) }\end{array}$ & $\begin{array}{l}\text { A TS melhora, moderadamente, a taxa de erradicação do } \\
H \text {. pylori em idade pediátrica (RR=1,14). Não obteve } \\
\text { superioridade em comparação com TT-10d }(p=0,24) \text { ou } \\
\text { TT-14d }(p=0,59) \text {. }\end{array}$ & 2 \\
\hline $\begin{array}{l}\text { Gatta et al., } \\
2009^{2}\end{array}$ & $\begin{array}{l}13 \mathrm{ECA} \\
n=3.006 \\
\text { adultos }+260 \\
\text { crianças e } \\
\text { adolescentes }\end{array}$ & $\begin{array}{l}\text { TS } 5 d+5 d \text { vs. TT- } 7 d \\
(11 E C A), T T-10 d(4 \text { ECA) } \\
\text { ou TT-14d (1 ECA) }\end{array}$ & $\begin{array}{l}\text { A TS mais eficaz na erradicação do } H \text {. pylori; } \\
\text { OR agrupado (TS vs. TT)=2,99; } \\
\text { OR (TS vs. TT, resistência à claritromicina) }=10,21 ; \\
\text { OR (TS vs. TT, na faixa pediátrica) }=1,98\end{array}$ & 1 \\
\hline $\begin{array}{l}\text { Jafri, } \\
\text { Hornung, } \\
\text { Howden, } \\
2008^{15}\end{array}$ & $\begin{array}{l}10 \text { ECA } \\
\text { (todos em } \\
\text { Itália) } \\
n=2.747\end{array}$ & $\begin{array}{l}\text { TS } 5 d+5 d \text { vs. TT-7d } \\
\text { (7 ECA), TT-10d (4 ECA) }\end{array}$ & $\begin{array}{l}\text { TS superior à TT standard } \\
\text { Taxa de erradicação global: TS=93,4\% vs. TT=76,9\%. Com a } \\
\text { TS, a redução do RR foi de } 71 \% \text { e do RA } 16 \% \text {. }\end{array}$ & 1 \\
\hline $\begin{array}{l}\text { Tong et al., } \\
2008^{20}\end{array}$ & $\begin{array}{l}11 \mathrm{ECA} \\
n=2.883\end{array}$ & $\begin{array}{l}\text { TS } 5 d+5 d \text { vs. TT-7d } \\
\text { (9 ECA), TT-10d (4 ECA) }\end{array}$ & $\begin{array}{l}\text { Taxa de erradicação da TS superior à TT-7d }(93,5 \% \text { vs. } 76,1 \% \text {; } \\
\text { RR=1,23) e à TT-10d }(92,4 \% \text { vs. } 79,2 \% ; R R=1,16) \text {. } \\
\text { Se resistência à claritromicina: } R R=2,01 \\
\text { Se resistência ao metronidazol: } R R=2,07\end{array}$ & 1 \\
\hline
\end{tabular}

Legenda: TT - Terapêutica Tripla; TS - Terapêutica Sequencial; ECA - Ensaio Clínico Aleatorizado e Controlado; RR - Risco Relativo; RA - Risco Absoluto; OR - Odds Ratio; ITT - Intention-to-treat analysis; PP - Per Protocol analysis

TT-7d (74,3\%), com um RR de 1,10 ( $p=0,0005)$. Não obstante, essa superioridade não se fez notar de forma estatisticamente significativa em comparação com a TT-10d e TT- $14 \mathrm{~d}$ ( $p=0,11$ e $p=0,73$, respetivamente). O metronidazol fez parte da TS em nove dos estudos e o tinidazol nos oito restantes. Ambos os regimes se mostraram superiores à TT (RR 1,10; $p=0,03$ e RR 1,09; $p=0,002$, respetivamente). Os au- tores concluíram que a TS parece ser superior à TT na erradicação da infeção por $H$. pylori, apesar de apresentar taxas de eficácia aparentemente inferiores nos países asiáticos. Os autores atribuem esses resultados à maior taxa de resistência concomitante à claritromicina e metronidazol presente nesses países, em relação a outros países da Europa, onde algumas MA prévias obtiveram taxas de erradi- 
cação de $91,0-93,5 \%{ }^{2,15,20-21}$

A MA de Chung et al., datada de 2013, incluiu seis ECA, todos com população adulta da Coreia (Quadro II). ${ }^{22}$ As taxas de erradicação agrupadas da TS (ITT=79,7\% e $P P=86,4 \%)$ revelaram-se superiores em relação à TT $(I T T=68,1 \% \mathrm{e}$ $P P=76,0 \%)$. O valor do $O R$ por análise $I T T$ e $P P$ foi de 1,84 e 1,97 , respetivamente, favorecendo a TS $(p<0,001)$. Os autores relataram também uma relação custo-eficácia favorável à TS, uma vez que a claritromicina, relativamente dispendiosa, é usada durante menos tempo nesse regime. Não houve diferenças significativas entre as duas terapêuticas quanto aos efeitos laterais. Estes resultados levaram os autores a concluir que a TS apresenta taxas de erradicação superiores às da TT, embora inferiores ao esperado e que, neste momento, é a opção terapêutica preferível. No entanto, consideram ser necessário o desenvolvimento de regimes terapêuticos mais efetivos.

A MA de Horvath, Dziechciarz e Szajewska, realizada em 2012, englobou 10 ECA, envolvendo 857 crianças dos 3-18 anos (Quadro II). ${ }^{23} \mathrm{~A}$ TS apresentou melhores taxas de erradicação do $H$. pylori em comparação com a TT-7d $(\mathrm{RR}=1,17 ; p=0,0006)$. A TS melhorou moderadamente a taxa de erradicação (78\% vs. $71 \%)$, sendo o NNT=15. Não houve diferenças significativas quanto à compliance ( $\geq 95 \%$ ) ou aos efeitos adversos entre ambos os regimes. Os autores concluíram que a TS pode ser considerada uma opção para aumentar a taxa de erradicação na faixa etária pediátrica, embora o seu benefício não seja tão evidente como o é na população adulta.

Na MA de Jafri, Hornung e Howden, ${ }^{15}$ publicada em 2008 (Quadro II), foram incluídos 10 ECA, todos realizados em Itália, embora, em dois deles, ${ }^{18-19}$ se tenham recrutado pacientes através de um site americano. Um dos estudos debruçou-se somente sobre a população pediátrica ${ }^{24}$ e outro utilizou ranitidina em substituição do IBP. ${ }^{25}$ Nenhum dos estudos comparou a TS com a TT-14d. A taxa de erradicação global da TS alcançou o valor de 93,4\%, enquanto a da TT registou 76,9\% de eficácia [redução do $\mathrm{RR}=71 \%$ e redução do Risco Absoluto (RA)=16\%]. A compliance foi semelhante em ambos os grupos (97,4\% na TS e $96,8 \%$ na TT), assim como a taxa de efeitos laterais. A TS foi superior à TT em todos os subgrupos analisados, nomeadamente presença de hábitos tabágicos, doença ulcerosa ou dispepsia não ulcerosa, resistência à claritromicina, imidazóis ou ambos, duração da TT, entre outros, levando os autores a concluir que a TS é superior em relação à TT de 7-10 dias na erradicação da infeção por $H$. pylori. Os autores alegaram igualmente que, a confirmar a superioridade da TS em relação à TT em futuros
ECA de boa qualidade, inclusivamente comparando com a TT-14d (amplamente utilizada nos EUA), a TS pode ser considerada o tratamento standard na erradicação do H. pylori.

A MA de Tong et al., de 2009 (Quadro II), incorporou 11 ECA, dos quais sete compararam a eficácia da TS com a TT$7 \mathrm{~d}$, dois com a TT-10d e outros dois com ambas as durações de TT. ${ }^{20} \mathrm{~A}$ TS foi associada a uma taxa de erradicação do H. pylori superior à TT-7d $(93,5 \%$ vs. $76,1 \%$; RR=1,23) $\mathrm{e}$ à TT-10d $(92,4 \%$ vs. $79,2 \%$; RR=1,16). A TS foi igualmente mais eficaz do que a TT nos doentes com dispepsia com úlcera péptica ou sem úlcera péptica $(R R=1,24$ e $R R=1,26$, respetivamente). A superioridade da TS tornou-se ainda mais evidente em indivíduos resistentes à claritromicina ( $R R=2,01)$ ou metronidazol $(R R=2,07)$. Não foram observadas diferenças significativas quanto aos efeitos colaterais. Os autores concluíram que a TS é um regime terapêutico promissor para a erradicação do H. pylori. Também nesta MA os autores sugerem a realização futura de mais ECA de boa qualidade, comparando diretamente TS com a TT-14d, de modo a averiguar qual deverá ser, de facto, o regime terapêutico de $1 .{ }^{\mathrm{a}}$ linha.

A RS de Zullo et al., realizada em 2012 (Quadro III), englobou 21 ECA, sendo um dos critérios de inclusão neste estudo a avaliação de um padrão modificado de TS, em comparação com a TT e TS convencionais. ${ }^{26} \mathrm{O}$ esquema modificado mais frequentemente utilizado incluía $7 d+7 d$ de um regime contendo tetraciclina, alcançando taxas de erradicação de $73,3 \%$, superiores aos $63,6 \%$ da TT-14d. A taxa de erradicação dos esquemas de TS modificada contendo levofloxacina $250 \mathrm{mg}$ ou $500 \mathrm{mg}$, 2id (>95\%) revelou-se superior, quer em comparação com a TS convencional contendo claritromicina $(80,8 \%)$ quer com a TT convencional $(71,7 \%)$. Um dos estudos constatou uma superioridade notória entre o esquema modificado, de 14 dias, contendo levofloxacina em relação à TT-14d $(86,6 \%$ vs. $45,3 \%) .{ }^{27}$ Os restantes esquemas modificados não alcançaram taxas de erradicação aceitáveis. Os autores concluíram que, tanto a TS modificada contendo levofloxacina como a TS modificada contendo tetraciclina, parecem ser mais efetivas na erradicação do H. pylori quando comparadas com a TT standard. No entanto, os autores alertam para o facto de a tetraciclina necessitar de ser administrada quatro vezes por dia neste esquema, podendo limitar a compliance do doente.

A outra revisão, de Kate et al., realizada em 2013 (Quadro III), incluiu 17 ECA, dos quais 12 reportaram melhores taxas de erradicação com a TS, um evidenciou melhores resultados com a TT e quatro não encontraram diferenças entre os dois regimes. ${ }^{1} \mathrm{O}$ maior ECA realizado na América La- 
tina ( $n=974$ ) foi um desses quatro estudos. ${ }^{28} \mathrm{Na}$ Europa foram realizados três estudos que constataram superioridade da TS em relação à TT, embora de forma estatisticamente significativa em apenas dois. ${ }^{29-30}$ Três ECA compararam a eficácia de ambas as terapêuticas em idade pediátrica $^{30-32} \mathrm{e}$ dois deles reportaram melhores taxas de erradicação com a TS, enquanto o outro ECA não demonstrou diferenças entre os regimes. Também em três estudos se efetuou uma análise de custos e, em todos, se demonstrou que a TS-10d era mais económica que a TT10d. . $7,33-34$ Quatro estudos compararam regimes de TS modificada-14d (dois contendo tetraciclina e dois contendo levofloxacina) com TT-14d e em todos se verificou superioridade estatisticamente significativa dos regimes de TS modificada-14d $(p<0,01)$. Não houve diferenças significativas em relação aos efeitos adversos, à exceção de um artigo. ${ }^{35}$ Os autores concluíram que a TS apresenta uma boa eficácia, aparenta ser mais económica e não condiciona um aumento nos efeitos adversos em relação à TT.

Relativamente aos ensaios clínicos, o de Liou et al., de 2013 (Quadro IV), publicado na Lancet, englobou 900 pacientes, com mais de 20 anos e com diagnóstico documentado de infeção por H. pylori (sem tratamento prévio), de seis diferentes clínicas de gastroenterologia tailandesas. ${ }^{36}$ As opções terapêuticas testadas consistiram em TS10d e TS-14d (lansoprazol 30mg + amoxilina $1 \mathrm{~g}$ na $1 .{ }^{\mathrm{a}}$ metade do esquema e lansoprazol 30mg + claritromicina $500 \mathrm{mg}+$ metronidazol 500mg na $2 .^{\mathrm{a}}$ metade do esquema, todos duas vezes por dia) e TT-14d (lansoprazol 30mg + amoxicilina $1 \mathrm{~g}$ + claritromicina $500 \mathrm{mg}$, todos duas vezes por dia). As taxas de erradicação foram de $90,7 \%$ para a TS$14 \mathrm{~d}, 87 \%$ na TS- $10 \mathrm{~d}$ e de $82,3 \%$ na TT- $14 \mathrm{~d}$. A TS- $14 \mathrm{~d}$ revelou-se superior à TT-14, quer pela análise ITT $(\mathrm{NNT}=12$; $p=0,003)$ quer pela análise $P P(\mathrm{NNT}=13,7 ; p=0,003)$. Não se observaram diferenças quanto aos efeitos adversos ou à compliance entre os três grupos. Os resultados obtidos levaram os autores a concluir que a TS-14d é superior à TT$14 \mathrm{~d}$ e deve ser recomendada como terapêutica de $1 .{ }^{a}$ linha na erradicação do H. pylori. De acordo com os critérios da escala SORT trata-se de um ECA de elevada qualidade, pelo que se atribui um NE 1.

O outro ECA, de Seddik et al., levado a cabo em 2013 (Quadro IV), envolveu 281 pacientes com infeção documentada por H. pylori, de um único centro hospitalar de Marrocos. ${ }^{37}$ Os pacientes foram randomizados para receber TS (omeprazol 20mg + amoxicilina lg nos primeiros cinco dias, seguido de omeprazol $20 \mathrm{mg}$ + claritromicina $500 \mathrm{mg}$ + tinidazol $500 \mathrm{mg}$ nos cinco dias subsequentes, todos duas vezes por dia) ouTT (omeprazol 20mg + amoxicilina 1g + claritromicina 500mg, durante sete dias, todos duas vezes por dia). A taxa de erradicação, por análise $I T T$ e $P P$, da TS $(82,9 \%$ e 89,9\%, respetivamente) demonstrou ser superior à TT $(65,9 \%$ e $71 \%$, respetivamente). Esta diferença foi estatisticamente significativa $(p 0,001)$, o que levou os autores a concluir que a TS-10dé mais efetiva que aTT na erradicação do H. pylori, sendo igualmente tolerada. De acordo com os critérios da escala SORT trata-se de um ECA de qualidade limitada, pelo que se atribui um NE 2 .

\section{CONCLUSÕES}

Esta revisão compreende um elevado número de estudos, a maioria de boa qualidade e com resultados consistentes, proporcionando robustez às conclusões retiradas pelos autores deste trabalho.

De acordo com a evidência disponível pode concluir-se que a TS é mais eficaz em comparação com a TT, sendo sobreponível em relação à compliance ou ocorrência de efeitos adversos, devendo ser considerada atualmente como terapêutica de $1 .{ }^{a}$ linha na erradicação do $H$. pylori (FR A).

Os estudos incluídos nesta revisão demonstraram que a TS é superior em relação à TT-7d e, somente em dois, não se comprovou o mesmo em relação à TT-10d. Quando comparada com a TT-14d, os resultados foram maioritariamente sobreponíveis. Estes resultados podem sugerir que o aumento da duração da TT para 14 dias seja suficiente para se alcançar a mesma taxa de sucesso obtida com a TS de 7-10 dias $^{3}$ embora os regimes de 14 dias da TS (7+7) apresentem superioridade terapêutica (FR B). Não obstante, o número de estudos a comparar a TS com a TT-14d é reduzido, pelo que não é possível tirar conclusões sólidas a este respeito. O prolongamento da TT para 14 dias abre, todavia, espaço à discussão de uma nova variante: a económica. $\mathrm{O}$ custo do tratamento é um importante fator a determinar o sucesso terapêutico, principalmente nos países em desenvolvimento. ${ }^{1}$ A TS revelou-se mais económica nos estudos em que foi realizada uma análise de custos. ${ }^{33-34}$ Uma vez que a claritromicina é o componente de custo mais elevado de entre os utilizados para erradicação do $H$. pylori, ${ }^{1}$ a redução da sua dose total presente na TS (cinco dias, 2id) é outro fator a favorecer este regime, em comparação com a TT ( $7,10 \mathrm{ou}$ 14 dias, 2id). Não obstante, em termos económicos latos, não podem ser retiradas ilações firmes, uma vez que os custos dependem também da taxa de sucesso/insucesso dos diferentes regimes e esse fator não foi contabilizado nestes estudos.

As principais NOC atuais - Maastricht IV, Asia-Pacific 


\begin{tabular}{|c|c|c|c|c|}
\hline Referência & $\begin{array}{l}\text { Estudos } \\
\text { Incluídos }\end{array}$ & Intervenção & Resultados & NE \\
\hline $\begin{array}{l}\text { Kate et al., } \\
2013^{1}\end{array}$ & $\begin{array}{l}17 \text { ECA } \\
n=4.169\end{array}$ & $\begin{array}{l}\text { TS } 5 d+5 d \text { vs. TT-7d } \\
\text { (8 ECA), TT-10d (2 ECA) } \\
\text { ou TT-14d (3 ECA) } \\
\text { TS 7d+7d (modificada } \\
\text { c/levofloxacina) vs. } \\
\text { TT-14d (2 ECA) } \\
\text { TS 7d+7d (modificada } \\
\text { c/tetraciclina) vs. } \\
\text { TT-14d (2 ECA) }\end{array}$ & $\begin{array}{l}\text { Dos } 17 \text { ECA, } 12 \text { reportaram melhores taxas de erradicação do } \\
\text { H. pylori com a TS, quatro não evidenciaram diferenças } \\
\text { significativas e um evidenciou superioridade com a TT Standard. } \\
\text { A TS modificada-14d também se revelou superior em comparação } \\
\text { com TT-14d }(p \leq 0,01) \text {. }\end{array}$ & 1 \\
\hline $\begin{array}{l}\text { Zullo et al., } \\
2012^{26}\end{array}$ & $\begin{array}{l}21 \mathrm{ECA} \\
n=4.169\end{array}$ & $\begin{array}{l}\text { TS modificada c/ } \\
\text { Tetraciclina vs. TS } \\
\text { modificada c/ quinolona } \\
\text { vs. TS standard vs. TT } \\
\text { standard }\end{array}$ & $\begin{array}{l}\text { As TS modificadas revelaram-se mais efetivas na erradicação do } \\
\text { H. pylori do que a TT; } \\
\text { TS-levofloxacina superior à TS conv. (95\% vs. 80,8\%). } \\
\text { Mais estudos são necessários para comparar a eficácia da } \\
\text { TS-standard com a TS-modificada. }\end{array}$ & 2 \\
\hline
\end{tabular}

Legenda:TT - Terapêutica tripla; TS - Terapêutica sequencial; ECA - Ensaio clínico aleatorizado e controlado.

\section{QUADRO IV. Ensaios clínicos controlados e aleatorizados}

\begin{tabular}{|c|c|c|c|c|}
\hline Referência & População & Intervenção & Resultados & NE \\
\hline $\begin{array}{l}\text { Liou et al., } \\
2013^{36}\end{array}$ & $\begin{array}{l}n=900 \\
\text { Ocultação da distribuição } \\
\text { Análise ITT (e PP) } \\
\text { Follow up >90\% }\end{array}$ & $\begin{array}{l}\text { TS } 5 d+5 d v s \text {. } \\
\text { TS } 7 d+7 d v s \text {. } \\
\text { TT } 14 d\end{array}$ & $\begin{array}{l}\text { A TS-14d revelou-se superior à TT-14, quer pela } \\
\text { análise ITT (NNT=12; } p=0,003) \text {, quer pela análise PP } \\
\text { (NNT=13,7; } p=0,003) \text {. } \\
\text { Sem diferença estatisticamente significativa entre } \\
\text { TS-10d e TT-14d. }\end{array}$ & 1 \\
\hline $\begin{array}{l}\text { Seddik et al., } \\
2013^{37}\end{array}$ & $\begin{array}{l}n=281 \\
\text { Ocultação não descrita } \\
\text { Análise ITT (e PP) } \\
\text { Follow up >90\% }\end{array}$ & TS $5 d+5 d$ vs. TT 7d & $\begin{array}{l}\text { A TS 10d é mais efetiva na erradicação do } H \text {. pylori em } \\
\text { comparação com a TT (ITT 82,8\% vs. } 65,9 \% \text {; } \\
\text { PP } 89,9 \% \text { vs. } 71 \% \text {; } P<0,001 \text { ) }\end{array}$ & 2 \\
\hline
\end{tabular}

Legenda: TT - Terapêutica tripla; TS - Terapêutica sequencial; NNT - Número necessário tratar; ITT - Intention-to-treat analysis; PP - Per Protocol analysis.

Guidelines e ACG - para a erradicação do H. pylori ainda recomendam a TT como terapêutica de $1 .^{\text {a }}$ linha a nível mundial. Todavia, é importante ressalvar que as últimas atualizações das mesmas datam de 2012, 2009 e 2007, respetivamente. Nas NOC de Maastricht e da ACG, a TS é recomendada como terapêutica alternativa, embora no primeiro caso essa indicação esteja restringida às áreas com taxas de resistência à claritromicina superior a $15-20 \%$, como é o caso de Portugal. Quer a Asia-Pacific Guidelines quer a ACG relataram a necessidade de validação da TS, através de mais estudos multicêntricos, randomizados e de boa qualidade, antes de poderem recomendar a TS

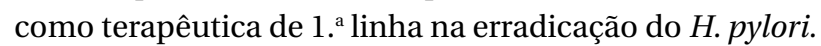
Entretanto, como reportado neste artigo, muitos ECA foram desenvolvidos desde essa altura, relatando taxas de erradicação da TS superiores à TT tradicional, o que poderá justificar uma futura revisão das recomendações pelos peritos.

Pelos dados obtidos por esta revisão, os autores realçam ainda dois pontos: o da evidência de superioridade da TS 
ser, aparentemente, maior quando se estudam populações em idade adulta e o facto de ser nas áreas de elevada resistência à claritromicina, metronidazol ou ambos, como é o caso de Portugal, que a superioridade da TS se demarca notavelmente face à TT (FR A). ${ }^{2,11,15,20}$

Não obstante, as taxas de erradicação de todos os regimes relatados são subótimas, uma vez que não alcançam sistematicamente valores de eficácia aconselháveis $(>90 \%)$ para doenças infeciosas. ${ }^{3,10,38} \mathrm{~A}$ procura constante por regimes terapêuticos mais efetivos ou por novos agentes para erradicar o H. pylori deve continuar. Tendo em conta que a resistência primária aos antibióticos se assume como a principal causa de falência terapêutica, ${ }^{39}$ a escolha do regime terapêutico deverá ser sempre baseada no conhecimento do padrão de resistência local, o qual necessita de ser permanentemente monitorizado. ${ }^{11}$ Novos regimes de terapêutica sequencial, modificada com levofloxacina ou tetraciclinas, têm sido testados, geralmente com sucesso, inclusivamente em comparação com a TT de 14 dias. ${ }^{26}$ No entanto, os estudos que testam esses regimes diferentes de TS são escassos e, portanto, não é possível, para já, tecer recomendações validadas.

Mais estudos de boa qualidade são necessários para avaliar qual o regime terapêutico mais vantajoso e se o principal fator de sucesso terapêutico está no regime ou na duração da terapêutica.

\section{AGRADECIMENTOS}

À Dra Mónica Oleastro, do Departamento de Doenças Infecciosas do Instituto Nacional de Saúde Dr. Ricardo Jorge, pelo contributo importante e cordialidade na cedência de artigos e dados estatísticos sobre o tema.

\section{REFERÊNCIAS BIBLIOGRÁFICAS}

1. Kate V, Kalayarasan R, Ananthakrishnan N. Sequential therapy versus standard triple-drug therapy for Helicobacter pylori eradication: a systematic review of recent evidence. Drugs. 2013;73(8):815-24.

2. Gatta L, Vakil N, Leandro G, Di Mario F, Vaira D. Sequential therapy or triple therapy for Helicobacter pylori infection: systematic review and meta-analysis of randomized controlled trials in adults and children. Am J Gastroenterol. 2009;104(12):3069-79.

3. Kim JS, Ji JS, Choi H, Kim JH. Sequential therapy or triple therapy for Helicobacter pylori infection in Asians: systematic review and meta-analysis. Clin Res Hepatol Gastroenterol. 2014;38(1):118-25.

4. Malfertheiner P, Megraud F, O'Morain CA, Atherton J, Axon AT, Bazzoli F, et al. Management of Helicobacter pylori infection: the Maastricht IV/ Florence Consensus Report. Gut. 2012;61(5):646-64.

5. Fock KM, Katelaris P, Sugano K, Ang TL, Hunt R, Talley NJ, et al. Second AsiaPacific Consensus Guidelines for Helicobacter pylori infection. J Gastroenterol Hepatol. 2009;24(10):1587-600.

6. Chey WD, Wong BC, Practice Parameters Committee of the American College of Gastroenterology. American College of Gastroenterology guideline on the management of Helicobacter pylori infection. Am J Gastroenterol.
2007; 102(8):1808-25.

7. Cabrita J, Oleastro M, Matos R, Manhente A, Cabral J, Barros R, et al. Features and trends in Helicobacter pylori antibiotic resistance in Lisbon area, Portugal (1990-1999). J Antimicrob Chemother. 2000;46(6):1029-31.

8. Megraud F, Coenen S, Versporten A, Kist M, Lopez-Brea M, Hirschl AM, et al. Helicobacter pylori resistance to antibiotics in Europe and its relationship to antibiotic consumption. Gut. 2013;62(1):34-42.

9. Laheij RJ, Rossum LG, Jansen JB, Straatman H, VerbeekAL. Evaluation of treatment regimens to cure Helicobacter pylori infection - a meta-analysis. Aliment Pharmacol Ther. 1999;13(7):857-64.

10. Vakil N. Helicobacter pylori treatment: a practical approach. Am J Gastroenterol. 2006;101(3):497-9.

11. Gatta L, Vakil N, Vaira D, Scarpignato C. Global eradication rates for Helicobacter pylori infection: systematic review and meta-analysis of sequential therapy. BMJ. 2013;347:f4587.

12. Laine L, Fennerty MB, Osato M, Sugg J, Suchower L, Probst P, et al. Esomeprazole-based Helicobacter pylori eradication therapy and the effect of antibiotic resistance: results of three US multicenter, double-blind trials. Am J Gastroenterol. 2000;95(12):3393-8.

13. Vakil N, Lanza F, Schwartz H, Barth J, et al. Seven-day therapy for Helicobacter pylori in the United States. Aliment Pharmacol Ther. 2004;20(1):99107.

14. Yoon H, Lee DH, Kim N, Park YS, Shin CM, Kang KK, et al. Meta-analysis: is sequential therapy superior to standard triple therapy for Helicobacter pylori infection in Asian adults? J Gastroenterol Hepatol. 2013;28(12):1801-9.

15. Jafri NS, Hornung CA, Howden CW. Meta-analysis: sequential therapy appears superior to standard therapy for Helicobacter pylori infection in patients naive to treatment. Ann Intern Med. 2008;148(12):923-31.

16. Kim JS, Kim BW, Ham JH, Park HW, Kim YK, Lee MY, et al. Sequential therapy for Helicobacter pylori infection in Korea: systematic review and metaanalysis. Gut Liver. 2013;7(5):546-51.

17. Ebell MH, Siwek J, Weiss BD, Woolf SH, Susman J, Ewigman B, et al. Strength of recommendation taxonomy (SORT): a patient-centered approach to grading evidence in the medical literature. Am Fam Physician. 2004;69(3):548-56.

18. Zullo A, Vaira D, Vakil N, Hassan C, Gatta L, Ricci C, et al. High eradication rates of Helicobacter pylori with a new sequential treatment. Aliment Pharmacol Ther. 2003;17(5):719-26.

19. Vaira D, Zullo A, Vakil N, Gatta L, Ricci C, Perna F, et al. Sequential therapy versus standard triple-drug therapy for Helicobacter pylori eradication: a randomized trial. Ann Intern Med. 2007;146(8):556-63.

20. Tong JL, Ran ZH, Shen J, Xiao SD, et al. Sequential therapy vs. standard triple therapies for Helicobacter pylori infection: a meta-analysis. J Clin Pharm Ther. 2009;34(1):41-53.

21. Zullo A, De Francesco V, Hassan C, Morini S, Vaira D, et al. The sequential therapy regimen for Helicobacter pylori eradication: a pooled-data analysis. Gut. 2007;56(10):1353-7

22. Chung JW, Ha M, Yun SC, Kim JH, Lee JJ, Kim YJ, et al. Meta-analysis: sequential therapy is superior to conventional therapy for Helicobacter pylori infection in Korea. Korean J Gastroenterol. 2013;62(5):267-71.

23. Horvath A, Dziechciarz P, Szajewska H. Meta-analysis: sequential therapy for Helicobacter pylori eradication in children. Aliment Pharmacol Ther. 2012;36(6):534-41.

24. Francavilla R, Lionetti E, Castellaneta SP, Magistà AM, Boscarelli G, Piscitelli D, et al. Improved efficacy of 10-Day sequential treatment for Helicobacter pylori eradication in children: a randomized trial. Gastroenterology. 2005;129(5):1414-9.

25. De Francesco V, Zullo A, Hassan C, Faleo D, lerardi E, Panella C, et al. Two 
new treatment regimens for Helicobacter pylori eradication: a randomised study. Dig Liver Dis. 2001;33(8):676-9.

26. Zullo A, De Francesco V, Hassan C, Ridola L, Repici A, Bruzzese V, et al. Modified sequential therapy regimens for Helicobacter pylori eradication: a systematic review. Dig Liver Dis. 2013;45(1):18-22.

27. Polat Z, Kadayifci A, Kantarcioglu M, Ozcan A, Emer O, Uygun A. Comparison of levofloxacin-containing sequential and standard triple therapies for the eradication of Helicobacter pylori. Eur J Intern Med. 2012;23(2):165-8.

28. Greenberg ER, Anderson GL, Morgan DR, Torres J, Chey WD, Bravo LE, et al. 14-day triple, 5-day concomitant, and 10-day sequential therapies for $\mathrm{He}-$ licobacter pylori infection in seven Latin American sites: a randomised trial. Lancet. 2011;378(9790):507-14.

29. Paoluzi OA, Visconti E, Andrei F, Tosti C, Lionetti R, Grasso E, et al. Ten and eight-day sequential therapy in comparison to standard triple therapy for eradicating Helicobacter pylori infection: a randomized controlled study on efficacy and tolerability. J Clin Gastroenterol. 2010;44(4):261-6.

30. Albrecht $P$, Kotowska M, Szajewska H. Sequential therapy compared with standard triple therapy for Helicobacter pylori eradication in children: a double-blind, randomized, controlled trial. J Pediatr. 2011;159(1):45-9.

31. Bontems P, Kalach N, Oderda C, Salame A, Muyshont L, Miendje DY, et al. Sequential therapy versus tailored triple therapies for Helicobacter pylori infection in children. J Pediatr Gastroenterol Nutr. 2011;53(6):646-50.

32. Erdur B, Ozturk Y, Gurbuz ED, Yilmaz O. Comparison of sequential and standard therapy for Helicobacter pylori eradication in children and investigation of clarithromycin resistance. J Pediatr Gastroenterol Nutr. 2012;55(5):530-3.

33. Valooran GJ, Kate V, Jagdish S, Basu D. Sequential therapy versus standard triple drug therapy for eradication of Helicobacter pylori in patients with perforated duodenal ulcer following simple closure. Scand J Gastroenterol. 2011;46(9):1045-50.

34. Zhou IQ, Xu L, Wang BF, Fan XM, Wu JY, Wang CY, et al. Modified sequen- tial therapy regimen versus conventional triple therapy for Helicobacter pylori eradication in duodenal ulcer patients in China: a multicenter clinical comparative study. Gastroenterol Res Pract. 2012;2012:405425.

35. Nadir I, Yonem O, Ozin Y, Kilic ZM, Sezgin O, et al. Comparison of two different treatment protocols in Helicobacter pylori eradication. South Med J. 2011;104(2):102-5.

36. Liou JM, Chen CC, Chen MJ, Chen CC, Chang CY, Fang YJ, et al., Sequential versus triple therapy for the first-line treatment of Helicobacter pylori: a multicentre, open-label, randomised trial. Lancet. 2013;381(9862):205-13.

37. Seddik H, Ahid S, El Adioui T, El Hamdi FZ, Hassar M, Abouqal R, et al. Sequential therapy versus standard triple-drug therapy for Helicobacter pylori eradication: a prospective randomized study. Eur J Clin Pharmacol. 2013;69(9):1709-15.

38. Vakil N, Megraud F. Eradication therapy for Helicobacter pylori. Gastroenterology. 2007;133(3):985-1001.

39. Hopkins RJ. In search of the Holy Grail of Heliocobacter pylori remedies. Helicobacter. 2001;6(2):81-3.

\section{CONFLITO DE INTERESSES}

Os autores declaram não ter conflitos de interesses.

\section{ENDEREÇO PARA CORRESPONDÊNCIA}

António Assunção Silva

E-mail: alpasilva18@gmail.com

Recebido em 16-06-2015

Aceite para publicação em 22-11-2015

Artigo escrito ao abrigo do novo acordo ortográfico.

\section{ABSTRACT}

\section{ERADICATION OF HELICOBACTER PYLORI:THE END OF THE LINE FOR STANDARD TRIPLE THERAPY?}

Objectives: To review the evidence for the effectiveness of triple therapy (TT) and sequential therapy (TS) in the eradication of Helicobacter pylori infection (H. pylori).

Data sources: MEDLINE and evidence-based medicine websites.

Methods: We conducted a search for clinical practice guidelines (CPG), systematic reviews (SR), meta-analyses (MA) and randomized clinical trials (RCT), published between June, 2004 and June, 2014, using the MeSH terms 'Helicobacter pylori', 'Drug theraPy' and 'Disease eradication'. The Strength of Recommendation Taxonomy (SORT) was used for the assignment of levels of evidence and the strength of recommendations.

Results: A total of 15 articles were selected out of 227 articles found. These included 3 CPG, 8 MA, 2 RS and 2 RCT. The CPG recommend TT as the preferred treatment. The $8 \mathrm{MA}$ and $2 \mathrm{RS}$ showed the superiority of ST in the eradication of Helicobacter pylori (ranging from 81 to $95.6 \%$ ) compared to the TT (for 7-10 days, consistently <80\%). The 2 EAC had overlapping findings with the MA and RS. Conclusions: TS has superior efficacy compared to TT and should be considered as the first line of therapy for eradication of $\mathrm{H}$. pylori infection (SOR A). When compared to TT for 14 days, conventional TS appears to have a similar rate of eradication. When given for more 14 days, TS is better than TT (SOR B). In areas of high resistance to clarithromycin, metronidazole or both, as in Portugal, TS is better than TT (SOR A).

Keywords: Helicobacter pylori; Drug Therapy; Disease Eradication. 